\title{
Grenze als Konfliktzone - Perspektiven der Grenzregimeforschung
}

\author{
Sabine Hess und Matthias Schmidt-Sembdner
}

\begin{abstract}
Der Beitrag skizziert verschiedene Konzepte von Grenzen und Grenzziehungsprozessen im Kontext der wissenschaftlichen Debatte um die sogenannte internationale (Flucht-)Migration. In Verbindung mit der Darstellung leitender Paradigmen der EU-europäischen Grenz- und Migrationspolitiken behandeln die Autor*innen die Perspektive der Autonomie der Migration und ihre methodologisch-theoretischen Implikationen für eine ethnografische Grenzregimeanalyse.
\end{abstract}

\section{Schlagwörter}

EU-europäisches Grenzregime, Schengen, ethnografische Grenzregimeanalyse, Autonomie der Migration, border struggles

\section{Die Allgegenwärtigkeit von Grenzen}

„Far from disappearing, many borders are being reasserted and remade through ambitious and innovative state efforts to regulate the transnational movement of people“ (Andreas/Snyder 2000, S. 2).

Diese Feststellung aus dem einflussreichen Werk The Wall around the West von Peter Andreas und Timothy Snyder (2000) war wohl nie so aktuell wie heute. Die Monate des Sommers 2015, als eine unerwartet hohe Anzahl an Fluchtmigrant*innen es schaffte, sich ihren Weg nach Westeuropa zu bahnen und hierbei buchstäblich die verschiedenen Grenzapparaturen ${ }^{1}$ (Walters 2002, S. 563) kraft ihrer Körper und des Begehrens nach einem besseren Leben zu überrennen, scheinen einer anderen Zeit anzugehören. Seitdem sind wir Zeug*innen einer unerwartet verstärkten Wiederkehr nationaler und regionaler Grenzapparaturen auf dem europäischen Kontinent in Gestalt von Zäunen, Gräben, Wachhunden und Wachtürmen (siehe dazu auch Leuenberger in diesem Band). Allerdings waren Zäune und Stacheldraht als Grenzinfrastrukturen nie gänzlich aus der europäischen Praxis verschwunden. Zwar wurden in den letzten zwei Jahrzehnten innerhalb Schengens Schlagbäume und andere materielle Grenzen weitgehend abgebaut, dafür wurden Zäune an der EU-Außengrenze bereits vor 2015 immer höher gezogen - sei es um die spanischen Exklaven Ceuta und Melilla oder entlang der griechisch-türkischen und der bulgarisch-türkischen Landesgrenzen -, die EU-Europa vom ,Rest trennen sollten, wie Stuart Hall und Bram Gieben (1992, S. 6) die postkoloniale Teilung der Welt beschrieben.

Neben diesen sichtbaren Grenzarchitekturen im Rahmen des EU-europäischen Rebordering wurde eine Vielzahl von technischen Apparaturen installiert, die mehr oder weniger ,unsichtbar' sind. Gelder in Millionenhöhe flossen in die Forschung und Entwicklung von computerge-

1 William Walters (2002, S. 563) verweist mit dem Begriff der Apparaturen auf die Fülle von polizeilichen und militärischen, aber auch kartografischen, diplomatischen, rechtlichen und geologischen Wissensformen und Praktiken, welche die Grenzen konstituieren. 
steuerten, digitalen und intelligenten Grenzüberwachungstechnologien. Die einstige Grenzlinie um das nationale Territorium herum hat netzwerkartigen Grenzinfrastrukturen Platz gemacht, in denen Satelliten, Drohnen und Radarsysteme zum Einsatz kommen und mit umfassenden Datenbanken vernetzt werden (vgl. Pötzsch in diesem Band). ${ }^{2}$ Bezugnehmend auf die Kontrolle des Mittelmeeres, beschreiben Sergio Carrera und Leonhard den Hertog (2015, S. 16) vom Center for European Policy Studies diese Entwicklungen als „surveillance race“, die nicht nur einen neuen militärisch-grenztechnologisch-industriellen Komplex hervorgebracht haben, sondern zu einer immensen Verräumlichung und Digitalisierung von Grenze führten. Diese Aufblähung und Multiplikation der Grenze ließ Etienne Balibar (2002, S. 84) bereits zu Beginn des neuen Jahrhunderts von einer „ubiquity of borders“ sprechen.

Die Allgegenwärtigkeit von Grenzen widerspricht der Hoffnung auf eine Welt obne Grenzen, die in den 1990er Jahren vom Ende des Kalten Krieges, dem Fall der als Eiserner Vorhang im antikommunistischen Sinne bezeichneten Grenze zwischen West- und Osteuropa sowie von der Erweiterung und Harmonisierung der Europäischen Union genährt wurde (vgl. Ohmae 1990). Trotz oder gerade aufgrund der Schaffung von „Schengenland“ als „Ort der Freiheit, der Sicherheit und des Rechts“ (vgl. Walters/Haahr 2004) und des allgemein fortschreitenden wirtschaftlichen, kulturellen und politischen Globalisierungsprozesses scheint diese Hoffnung der Vergangenheit anzugehören (vgl. Newman 2006; Donnan/Wilson 2010, S. 2).

Was den sozial- und kulturwissenschaftlichen Forschungsgegenstand der Grenze betrifft, betonte David Newman bereits 2006 in seinem Überblicksartikel über Konzepte und Ansätze im interdisziplinären Feld der Border Studies, dass Grenzforschung zu einer wachsenden Industrie geworden sei (vgl. Newman 2006, S. 144). Auch wenn das nicht auf den deutschsprachigen wissenschaftlichen Kontext zutrifft - bis heute existieren nur sehr wenige institutionelle Forschungskontexte mit dieser Bezeichnung -, können wir dennoch beobachten, dass eine gewisse „Explosion“ von Studien und Forschungsprojekten über Grenzen stattgefunden hat, wie Hastings Donnan und Thomas M. Wilson es 2010 in ihrem Sammelband Borderlands: Ethnographic Approaches to Security, Power, and Identity ebenfalls feststellen. Nicht nur in den Border Studies, sondern auch im weiteren Feld der Migrations- und Mobilitätsstudien wird die neue und wachsende Bedeutung der Grenzthematik deutlich. So steht auch in dem im Jahr 2013 erschienen Artikel Regimes of Mobility across the Globe von Nina Glick Schiller und Noel B. Salazar die Frage von Grenzziehungsprozessen im Vordergrund. Auch sie beobachten eine zunehmende Wiederkehr nationaler Grenzen und ethnischer Grenzziehungsprozesse inmitten globaler Wirtschaftskrisen und sehen ein „single global mobility regime“ im Entstehen:

„Oriented to closure and to the blocking of access, premised not only on ,old“ national or local grounds but on a principle of perceived universal dangerous personhoods [...] In practice, this means that local, national, and regional boundaries are now being rebuilt and consolidated“ (Glick Schiller/Salazar 2013, S. 199).

Doch wie werden in den Border Studies, die sich mit internationalen Migrationsbewegungen und Grenzziehungsprozessen auseinandersetzen, Grenzen konzeptualisiert? Welches Verständnis von Mobilität und Bewegung vis-à-vis der Grenze liegt diesen Konzepten zugrunde oder

2 Darunter Spaniens integriertes System zur Außenüberwachung (Sistema Integrado de Vigilancia Exterior, SIVE), das 2002 eingeführt wurde, das meergestützte Überwachungssystem (MARSUR) von 2005 und das Europäische Grenzüberwachungssystem (EUROSUR), das von der EU 2013 neben großen Datenbanken wie der Fingerabdruckdatenbank Eurodac, dem Schengener Informationssystem (SIS) sowie dem Visa-Informationssystem (VIS) eingeführt wurde. 
ergibt sich daraus? Und wie kann daraus der Begriff des Grenzregimes abgeleitet werden, wie er im Rahmen der kritischen Migrations- und Grenzregimeforschung über die letzten 10 Jahre entwickelt wurde?

Beginnend mit einer Diskussion verschiedener konzeptioneller Entwürfe von Grenze und ihrer Funktion werden wir nachfolgend die zugrunde liegenden Konstruktionsmomente und Paradigmen des europäischen Grenzregimes aufzeigen. Daran anschließend werden wir mit dem Konzept der Autonomie der Migration als Prisma oder Perspektive für eine Theoretisierung der Grenze plädieren, die Grenze strukturell als Konfliktzone versteht, und skizzieren den Ansatz der ethnografischen Grenzregimeanalyse und seine methodologisch-theoretischen Implikationen.

\section{Grenze als Barriere, Tor und/oder Transformationsregime?}

David Newman (2006, S. 145) betont, dass es nicht die eine Grenztheorie gäbe. Allerdings herrscht im interdisziplinären Feld der Border Studies mittlerweile ein weitgehender Konsens bezüglich der Transformationsprozesse, denen territoriale Staatsgrenzen in den letzten zwei Jahrzehnten unterlagen - bezüglich ihrer Form, Territorialität bzw. Verräumlichung, ihrer Rolle und Funktion sowie hinsichtlich ihrer Hervorbringung, Performanz und ihres Regierens (vgl. Donnan/Wilson 2010). In diesem Sinne besteht größtenteils Einigkeit darin, dass Staatsgrenzen nicht länger als statische Linien oder Abgrenzungen eines souveränen Staates und nationalstaatlicher Macht konzeptualisiert werden können, wie in dem Manifest Lines in the Sand: Towards an Agenda for Critical Border Studies (Parker/Vaughan-Williams 2009) nachdrücklich betont wird. Grenzen sind daher weniger denn je als ,Rand des Politischen` zu verstehen, sondern sind „zu Objekten, genauer gesagt zu Dingen im Raum des Politischen“ geworden, wie es Étienne Balibar (1997/2006, S. 250, Herv. i. O.) schon früh formulierte.

Diese Einsicht in den Wandel der Gestalt und Funktion der Grenze führte dementsprechend auch zu einer geografischen Neufokussierung der Forschung weg von der Staatsebene hin zu Regionen, Kommunen oder Gated Communitys sowie zur transnationalen oder globalen Ebene (vgl. Laine 2016). Die wahrgenommenen Transformationen ebneten auch den Weg für eine methodologische Neuausrichtung. „Statt die Grenze an sich“ (Newman 2006, S. 144) zu fokussieren, rückten zunehmend Prozesse der Grenzziehungen und Praktiken des borderings in den Mittelpunkt der Betrachtung. Nichtsdestotrotz konzeptualisieren die meisten Studien die Grenze selbst als eine „Exklusions- und Schutzbarriere“, wie etwa auch Hastings Donnan und Thomas M. Wilson (2010, S. 11) in ihren Forschungen zu täglichen Grenzüberschreitungspraktiken von „Grenzbewohner*innen“. Im Foucault'schen Sinne werden Grenzen in diesem Zusammenhang als Ordnungstechnologie gesehen, die uns von denen, das Hier vom Dort und das Innen vom Außen unterscheidet, wie Henk van Houtum und Ton van Naerssen in ihrem Artikel Bordering, Ordering and Othering (2002) beschreiben. Diese Vorstellung von Grenzen als Grenzen ziehende Technologien lädt zu einem weiten metaphorischen Gebrauch des Begriffs der Grenze im deterritorialen und eher sozialen Sinne ein, welcher Frederik Barths (1969/1998) Konzept der (sozialen und ethnischen) Grenzziehung (boundary) sehr nahekommt (vgl. auch Höfler/Klessmann in diesem Band).

Dabei war auch die territoriale Staatsgrenze immer mehr als nur eine Linie auf dem Boden, manifestiert durch eine materielle Infrastruktur. Grenzen mussten schon immer auch performiert werden und benötigten in diesem Sinne weitere soziale und kulturelle Komponenten, 
Investitionen und Arrangements. So belegt die Geschichtsforschung klar, wie schwierig es war, insbesondere die nationalen Grenzen zu errichten und Grenzpraktiken durchzusetzen (vgl. François et al. 2007). Grenzen benötigen nicht nur Gesetze, Bürokratien, Abläufe und Repräsentationen (Pässe, Stempel, Akten, Landkarten), sondern müssen auch ihren Platz auf unseren geistigen Landkarten, in kulturellen Bildern und unseren moralischen Urteilen haben, um „bedeutungsgebend und bedeutungstragend“ zu sein (Donnan/Wilson 2010, S. 4). In seinem wegweisenden Artikel Mapping Schengenland: Denaturalizing the Border skizziert William Walters (2002) drei historische Typologien von Grenze: die „geopolitische“, die „nationale“ und die „biopolitische Grenze“. Hier zeigt er auf, dass selbst die geopolitische Grenze - als Ausdruck des klassischen Verständnisses des 18. und 19. Jahrhunderts von Nationalstaaten als territorial definierte Einheiten und der entsprechenden territorialisierten Grenze als Linie, an der sich die Streitkräfte versammelten - als Assemblage betrachtet werden muss: „There is a whole apparatus connected with the geopolitical border - not just a police and military system, but cartographic, diplomatic, legal, geological, and geographical knowledges and practices“ (ebd., S. 563). Walters spricht von Grenzen als eine „Kunst des Regierens“, während der Kolonialzeit war die Grenzziehungspraxis dann vor allem eine Kunst des imperialen Regierens (ebd., S. 564).

Da sich die wenigsten Grenzen als totalitäre ,Exklusionsbarriere' begreifen lassen, müssen wir mit der Vorstellung eines antithetischen Verhältnisses zwischen Grenzen und Mobilität brechen. So war die Grenze auch immer ein Tor und in diesem Sinne eine Mobilitätsinstitution (vgl. Donnan/Wilson 2010). Grenzen unterscheiden sich in Bezug auf diese Flow-ManagementCapacity und ihre Filterfunktion jedoch erheblich, nicht nur in Bezug darauf, Ströme zu verlangsamen bzw. zu beschleunigen, sondern auch, wie sie selektiv auf Mobilitäten zugreifen. ${ }^{3}$ Sie werden daher auch sehr unterschiedlich erfahren. Der US-amerikanische Kulturanthropologe Michael Kearney äußerte sich zu dieser Funktion der Grenzen in seiner Forschung über die seit Langem militarisierte US-amerikanisch-mexikanische Grenze bereits in den 1990er-Jahren wie folgt:

„Rhetoric aside, [...] the de facto immigration policy of the unitedstatesian government is not to make the US-Mexican border impermeable to the passage of ,illegal' entrants, but rather to regulate their flow, while at the same time maintaining the official distinctions between [...] kinds of people, that is to constitute classes of peoples“ (Kearney 1991, S. 58).

Kearney geht dabei noch einen Schritt weiter als nur von der filternden Funktion der Grenzkontrolle zu sprechen, „that separates out the unwanted from the wanted cross-border flows“ (Andreas 2000, S. 4). Vielmehr verweist Kearneys Formulierung, dass die Grenze ,Klassen von Menschen' bildet, darauf, dass Grenze nicht auf ihre Funktion als repressives Instrument reduziert werden kann, sondern eher als ,produktiver' Mechanismus im Sinne von Foucaults Begriff der Biomacht (vgl. Lemke 2007) zu verstehen ist. Dies bringt später William Walters (2002) mit seinem Begriff der „biopolitischen Grenze“ explizit zum Ausdruck. Auch Sandro Mezzadra und Brett Neilson (2013; in diesem Band) folgen in ihrer Publikation Border as

3 Auch Peter Andreas (2000, S. 4) weist in Bezug auf die europäische Außengrenze auf ihre Permeabilität hin und wendet sich gegen weitverbreitete gesellschaftliche Vorstellungen, die insbesondere ihre Schließungsfunktion adressieren, wie sie in der Metaphorik der Festung Europa zum Ausdruck kommen. In diesem Zusammenhang beschreibt Chris Rumford (2008, S. 3) Grenzen als „asymmetrische Membrane[n]“ und William Walters (2006, S. 197) verwendet die Metapher einer „firewall“, die basierend auf einem sehr differenzierten Raster ablehnt und selektiert. 
Method dieser Perspektivierung und sprechen von der „differentiellen Inklusion“, womit sie den Blick auf die differenzierende und hierarchisierende Funktion von Grenze lenken, die die Subjekte im politischen Raum in verschiedene Formen der Unterordnung, Diskriminierung und Segmentierung bringt. Lydia Morris (2002) spricht in dieser Hinsicht von der „civic stratification“. Die Grenze kann in diesem Verständnis als Transformationsregime von Rechten und Status verstanden werden. Der rechtlich-politische (staatsbürgerliche) Status der Menschen wird zum Zeitpunkt seiner Grenzüberschreitung in Frage gestellt oder gar beseitigt, indem die grenzüberschreitende Mobilität einmal als ,illegal' oder ,irregulär' delegitimiert wird und dem dazugehörigen Subjekt seine bisherigen Rechte entzogen werden, während andere Mobilitäten mehr oder weniger unbefragt als Tourist"innen, Fachkräfte oder Expert*innen reisen und als volle Rechtsubjekte weiter handeln können. In diesem Sinne stellt die Grenze ein gigantisches Transformationsregime dar, das durch die kategoriale Erfassung und Bearbeitung der Mobilitäten neue Hierarchien von Menschen unter den migrationspolitischen Kategorien entstehen lässt.

Diese biopolitische Funktion der Grenze, das zeigt William Walters (2002) weiter, verändert jedoch auch ihre Form und Gestalt. Wenn sich bereits die geopolitische Grenze nicht als Linie verstehen lässt, wie wir gezeigt haben, so muss man sich die biopolitische Grenze eher als ,Maschine، vorstellen, wie es Walters beschreibt, oder als ein Regime bestehend aus einem Gefüge aus neuen und alten, einfachen und komplexen Technologien, Infrastrukturen, Gesetzen, Institutionen und Diskursen: „These include passports, visas, health certificates, invitation papers, transit passes, identity cards, watchtowers, disembarkation areas, holding zones, laws, regulations, customs, and excise officials, medical and immigration authorities“ (ebd., S. 572).

Dabei macht Walters auf eine weitere Funktion der biopolitischen Grenze aufmerksam und hebt hervor, dass die Grenze zu einem ,privilegierten Ort' wurde, an dem politische Behörden biopolitische Kenntnisse über Bevölkerungen erlangen können: „In this sense the border actually contributes to the production of population as a knowable, governable entity" (ebd., S. 573).

Eine derartig ausgerichtete biopolitische Grenze hat neue technopolitische Verräumlichungen hervorgebracht, wie sie mit Begriffen wie border zones, borderlands oder borderscapes umschrieben werden. Gleichzeitig beinhalten diese Konzepte Ideen mobiler, veränderlicher, selektiver und differenzierter Grenzsituationen. Es wird auch von „mobile borders“ (Kuster/Tsianos 2014, S. 3) oder „networked borders“ (Rumford 2006, S. 157) gesprochen. In diesem Zusammenhang argumentiert Balibar (2002, S. 78f.) für eine analytische Fassung der Grenzen als überdeterminiert, heterogen und polysemisch - „that is to say that borders never exist in the same way for individuals belonging to different social groups" (ebd., S. 79). Diejenigen, die die entsprechenden wirtschaftlichen Mittel, Nationalität und Dokumente besitzen, haben die europaweite Reisefreiheit in den letzten Jahren genossen. Andere hingegen, wie die Menschen, die zu den Staaten aus dem Globalen Süden gehören, begegnen den Grenzen in Zügen oder an Bahnhöfen, auf Flughäfen, in Schulen und in Gesundheitseinrichtungen auf Gemeindeebene.

Ein solches Verständnis von Grenzen führte zunehmend zu einer stark praxeologischen Ausrichtung der Border Studies, wobei nun Prozesse von doing und performing borders interessieren (Houtum/Naerssen 2002, S. 126; Salter 2011; siehe auch Wille in diesem Band). So wird die Grenze als ein Effekt einer Vielzahl von Akteur*innen und Praktiken verstanden, was Chris Rumford (2008) mit dem Konzept des borderwork explizit aufgreift (vgl. auch Salter 2011). Hiermit lenkt er die Aufmerksamkeit auf die alltäglichen Mikropraktiken der an 
der Grenzproduktion beteiligten Politiker*innen, Grenzbeamt*innen, Journalist*innen, Wissenschaftler*innen, Richter*innen, NGO-Mitarbeiter*innen, von Transportpersonal und den vielen anderen, die die Grenze immer wieder neu interpretieren und ausagieren müssen, um sie in Kraft zu setzen. In den vergangenen Jahren wurde dieses Konzept im Sinne neuerer materialitätstheoretischer Ansätze sowie im Kontext der Infrastrukturstudien erweitert, um die Aufmerksamkeit auch auf das wachsende Arsenal von Technologien wie Drohnen, Satelliten, Wärmebildkameras, Scannern und Datenbanken zu ziehen. Aus dieser Perspektive wird die Grenzziehung als performativer Akt verstanden. Aufbauend auf Judith Butlers (1991) Begriff der Performativität weist Marc Salter auf die Tatsache hin: „Sovereignty, like gender, has no essence, and must continually be articulated and rearticulated in terms of ,stylized repetition of acts' of sovereignty" (Salter 2011, S. 66).

\section{Schengen als Laboratorium: Paradigmen des europäischen Grenzregimes}

Die Betonung der skizzierten Transformation der Grenze von einer Demarkationslinie rund um ein Staatsgebiet hin zu einer allgegenwärtigen, technisch-sozialen, deterritorialisierten Apparatur oder einem Regime, welches geografisch ausgedehnte Grenzräume (borderscapes) bildet (vgl. Brambilla 2015), ${ }^{4}$ stellt - wie ausgeführt - den gemeinsamen Nenner der Forschungen in den internationalen Border Studies dar, welche den Zusammenhang von Grenze und der Regulation grenzüberschreitender Mobilitäten von Gütern oder Menschen adressieren. Das gilt vor allem für die EU, die - wie Sabine Hess und Bernd Kasparek (2017) im Artikel Deand Restabilising Schengen: The European Border Regime after the Summer of Migration darlegen - als ,Labor' besagter Transformation betrachtet werden kann. Mit dem Schengener Abkommen von 1985 läutete das europäische Projekt mit dem neu geschaffenen Begriff der Außengrenze als zentralem Mechanismus und Raum für Migrationskontrolle die Bildung eines europäischen Grenzregimes ein. Obwohl dieses Projekt vorerst außerhalb des offiziellen EG/ EU-Rahmens stattfand, war dieser weltweit einzigartige Prozess der Regionalisierung und der supranationalen Harmonisierung eine treibende Kraft für einen beschleunigten und vertieften Prozess der Europäisierung, der im Vertrag von Amsterdam (1999) und später im Vertrag von Lissabon (2009) gipfelte.

Dabei lässt sich die Herausbildung des europäischen Grenzregimes nicht als ein linearer politischer Akt verstehen, vielmehr ist er gekennzeichnet von zahlreichen Rückschlägen und widersprüchlichen (politisch-sozialen) Visionen und Interessen unterschiedlichster Akteursgruppen (vgl. Hess/Tsianos 2007; Karamanidou/Kasparek 2018). Die verschiedenen Orte, Akteure, Entwicklungspfade und Tempi seiner Hervorbringung führen jedoch auch dazu, dass das europäische Grenzregime als eine multiskalare Assemblage zu verstehen ist: bestehend aus Agenturen der EU wie FRONTEX (Europäische Grenz- und Küstenwache), Institutionen des europäischen Rechts (wie das Gemeinsame Europäische Asylsystem) und Standardisierungs- und Harmonisierungsprozessen innerhalb der EU, vor allem im Bereich des Grenzmanagements (,Integriertes Grenzmanagement' genannt). Zu ihr gehört auch ein wachsender militärisch-industriell-wissenschaftlicher Komplex, der hauptsächlich von der EU gefördert wird, und sich neben traditionelleren politischen nationalen Apparaten der Migrationskontrolle, die sich seit den 1970er-Jahren entwickelten, einreiht. Ein weiteres Merkmal ist die flexible Beteiligung

4 Chris Rumford (2006, S. 159) spricht von einem „Zustand der Post-Territorialität“. 
von IGOs (Intergovernmental Organizations), darunter internationale und zwischenstaatliche Organisationen wie der Hohe Flüchtlingskommissar der Vereinten Nationen (UNHCR) oder die Internationale Organisation für Migration (IOM).

Ferner ist dem europäischen Grenzregime von Anfang an ein grundlegendes Kontrolldilemma des EU-Schengenlands eingeschrieben, auf welches Gallya Lahav und Virginie Guiraudon (2000) in ihren frühen Ausführungen hinweisen. So ist mit der Errichtung des EU-Binnenmarktes die dilemmatische Frage zentral gestellt worden, wie sich ein neoliberales, wirtschaftliches Paradigma des (möglichst weltweiten) freien Güter-, Dienstleistungs- und Kapitalverkehrs mit dem kontinuierlichen biopolitischen Wunsch vereinbaren lässt, den Personenverkehr und die (nationalen) Bevölkerungen zu kontrollieren. Während die EU mit dem Vertrag von Amsterdam sich zunehmend als politischer harmonisierter Raum konstituierte und die Mobilität ihrer Bürger*innen zunehmend binneneuropäisch absicherte, hat sie mit der Konstruktion der sogenannten ,Drittstaatsangehörigen' eine neue Differenzierung geschaffen und dem weltweiten freien Personenverkehr eine Absage erteilt.

Viele Autor*innen der Grenzforschung und der Europaforschung machen darauf aufmerksam, dass die Schaffung des EU-Binnenmarktes einem breiten Feld an Sicherheitsakteuren die Tür öffnete und zu einer verschärften Versicherheitlichung von Fragen der Mobilität führte (vgl. Huysmans 2000; Bigo/Guild 2005). William Walters und Jens Henrik Haahr (2004, S. 95) meinen gar, dass „Schengenland can be seen as having certain acts of securitisation as its conditions of possibility“. Darüber hinaus bestand die wesentliche praktische Antwort des Grenzregimes auf das Kontrolldilemma laut Lahavs und Guiraudons (2000) darin, die Grenzkontrollen fernab der Grenze und außerhalb der Staaten zu platzieren, was zu der bereits skizzierten Verräumlichung und geografischen Ausdehnung der Grenze führte. Dabei bestand die Vision der Europäischen Kommission in einer smarten, technisch-wissenschaftlichen, unsichtbaren und doch selektiven Grenze, die zwischen erwünschten Reisenden und unerwünschten Migrant*innen unterscheiden kann (Commission of the European Communities 2008).

In diesem Kontext haben sich vier Paradigmen, die das europäische Grenzregime leiten und kennzeichnen, herausgebildet. Als erstes ist das Paradigma der remote control und der Externalisierung zu nennen. Neben der geografischen Ausdehnung führte dieses auch zu einer unvorhergesehenen Multiplikation und Diversifizierung der Akteur*innen: von staatlichen zu nichtstaatlichen, von internationalen als auch von auf lokaler Ebene auftretenden Akteur*innen (vgl. Lahav/Guiraudon 2000; Lavenex 2004; Zolberg 2006; Hess/Tsianos 2007; Bialasiewicz 2012). Das zweite Paradigma ist, wie bereits angedeutet, das einer ,robusten " und doch aufgrund von Technologisierung, Digitalisierung und Biometrisierung smarten Außengrenze (vgl. Koslowski 2005; Broeders 2007; Dijstelbloem et al. 2011; Kuster/Tsianos 2014). Diese zwei Dimensionen sind im Rahmen der Border Studies grundlegend analysiert worden. Ein drittes Paradigma schuf ein internes Regime, das die Institution des Asyls durchdrungen hat. Zentral sind hier die Dublin-Verordnungen und die Eurodac-Bestimmungen ${ }^{5}$ zu nennen. Sie zielen auf die Immobilisierung der Migrationsbevölkerung ab, welche im Rahmen des europäischen Asylsystems Schutz sucht. Mit Hilfe der ersten paneuropäischen Fingerabdruckdatenbank Eurodac im Zusammenspiel mit der aktuellen Fassung Dublin-III-Verordnung wird der Zugang zu den nationalen Asylsystemen für Fluchtmigrierende stark beschränkt. Mit einer Vielzahl von Kriterien regelt das Dublin-System, welcher Mitgliedsstaat für die Durchführung

5 Der Begriff Eurodac ist ein Akronym, das sich aus „automated European dactylographic system“ ableitet. 
eines Asylverfahrens zuständig ist. Als zentrales Kriterium hat sich dabei das Land der ,ErstEinreise ${ }^{6}$ herausgestellt. Eine Weiterwanderung in einen anderen europäischen Mitgliedstaat führt zur Einleitung eines Dublin-Verfahrens, das in den meisten Fällen mit dem staatlichen Versuch einer Abschiebung in den Ankunftsstaat einhergeht (vgl. Schuster 2011; Borri/Fontanari 2016; Kasparek 2016).

Das vierte und letzte Paradigma, das vor allem in den Jahren vor 2015 hervortrat, ist eine ansteigende Humanitarisierung ${ }^{6}$ der Grenze, von Walters (2011) beschrieben als die „Geburt der humanitären Grenze“. Diese Entwicklung wurde angesichts der wachsenden Anzahl von tödlichen Schiffbrüchen und Tragödien im Mittelmeer in den Jahren 2013 und 2014 beschleunigt, als das Überqueren der Grenze offenkundig zu einer Sache von Leben und Tod wurde. Der humanitäre Diskurs geht jedoch zurück auf einen Brief des ehemaligen britischen Premierministers Tony Blair an seinen griechischen Amtskollegen Costas Simitis im März 2003. Griechenland hatte zu dieser Zeit die EU-Ratspräsidentschaft inne und Blair warb mit dem im Brief beigefügten Plan New Approaches to Asylum Processing and Protection ${ }^{7}$ für eine Diskussion auf EU-Ebene um ein „besseres globales Management von Asylprozessen“. Mit seinen Vorschlägen zur Vorverlagerung der Grenz- und Migrationskontrolle über das EU-Territorium hinaus wird es in erster Linie als Gründungsdokument für die Externalisierungspolitik der EU gelesen. Es ist jedoch mehr als das: Der Plan macht sich auch eine stark humanitäre Rhetorik zunutze und - wir würden sogar sagen - instrumentalisiert sie, um die weitere Externalisierung und Verschärfung der Grenzkontrollen als humanitäre Geste des Schutzes zu legitimieren.

Auch im Rahmen unseres ersten Transit-Migration-Forschungsprojekts in den frühen 2000erJahren konnten wir Prozesse beobachten, die wir als NGOization und Gouvernementalisierung der Politik bezeichnet haben. Wir wiesen in diesem Zusammenhang darauf hin, dass die Expansion des Grenzregimes weit über die Außengrenzen der EU hinaus nicht nur durch Sicherheitsakteur*innen stattfand, sondern vor allem auch durch einen speziellen Verweis auf und die Artikulation von humanitären Positionen und Praktiken, wie sie insbesondere im Bereich der Anti-trafficking-Politik und im Kontext des asylpolitischen Feldes kenntlich wurden (vgl. Hess/Karakayali 2007). Nach dem Tod von mehr als 600 Migrant*innen bei einem Schiffsunglück 2013 vor Lampedusa wurde der humanitaristische Diskurs gänzlich dominant. Dieser Paradigmenwechsel - von einer Politik des Sterbenlassens und der Push-Backs hin zu einer Politik des Rettens - schien aufgrund weitreichender Veränderungen möglich zu sein, die zum Teil auch durch unaufhörliche Kämpfe der Migration, transnationale Solidaritätsnetzwerke und die professionalisierten kritischen Wissenspraktiken der NGOs sowie rechtliche Interventionen entstanden sind. Diese haben zu einer weiteren Verrechtlichung des Grenzregimes und zu einer gewissen Stärke menschenrechtlicher Ansätze geführt (vgl. Hess 2016a).

6 Wir halten uns an William Walters (2011) und Didier Fassins (2007) Konzeption der Humanitarismus, unter der beide mehr als nur „Ideen und Ideologien“ oder „einfach nur die Aktivität gewisser nichtstaatlicher Akteure“ verstehen. Sie begreifen Humanitarismus als eine spezielle Form des Regierens, als eine Rationalität der Macht und platzieren die Debatte „in Relation zur Regierungsanalytik“ (Walters 2011, S. 143). Wie Paolo Cuttitta (2016) es ausdrückt, führt dies zu einer speziellen operationalen Logik, die ihren Ausdruck in einem zunehmend organisierten und internationalisierten Versuch findet, das Leben der verwundbarsten Bevölkerungen der Welt zu retten, deren Wohlergehen zu steigern und deren Leiden zu lindern - und das nun auch im Bereich der Grenzkontrolle.

7 www.statewatch.org/news/2003/apr/blair-simitis-asile.pdf, 14.02.2020. 


\section{Umkämpfte Grenzen und die konstitutive Macht der Migration}

Die Migrationsbewegungen des Sommers 2015 haben das Grenzregime mit seinen hier dargelegten vier zentralen Paradigmen in seinen Grundfesten erschüttert und Kerninstrumente wie das Dublin-System seitdem paralysiert (vgl. Schmidt-Sembdner 2019). Die Ereignisse haben die Mächtigkeit und die agency der Migration nicht nur zurück auf die politische Agenda gesetzt, sondern auch die Border Studies epistemologisch herausgefordert. Selbst wenn die darin zum Ausdruck kommenden Ansätze praxeologisch argumentierten und die borderwork - und damit die praktische Involviertheit verschiedener Akteur*innen in der Hervorbringung von Grenzen (vgl. Pallister-Wilkins 2017, S. 6) - in den Mittelpunkt der Betrachtung stellten, wurde die Migration selbst dabei nur marginal in den Blick genommen. Die Migration wird, wenn, dann als Objekt und Adressat der Grenze verhandelt, nicht jedoch als Kraft und (ko)konstitutiver Faktor politischer Transformationen und der umkämpften Aushandlung und Ausdeutung von Grenzen. Damit wird die strukturelle Dominanz der Migrationskontrollapparate epistemologisch reproduziert und ein geteiltes Geschehen der Migration konstruiert: auf der einen Seite steht ein mehr oder weniger monolithischer Apparat, welcher die Migration aufhalten, ausschließen, unterdrücken und ausbeuten will. Auf der anderen Seite gelten Migrant*innen als ,passive Opfer', Migration wird individualisiert (vgl. Papadopoulos et al. 2008, S. 3) und ihre Formen des sozialen Handelns, die politischen und sozialen Konflikte, die sie eröffnen (vgl. Bojadžijev 2006, S. 140), bleiben unberücksichtigt. Die Viktimisierung der Migrant*innen wird aber auch durch Advocacy-Gruppen verstärkt, die sich für Migrant*innen einsetzen und dazu tendieren, deren Ohnmacht hervorzuheben (vgl. Karakayali 2008).

Im Gegensatz dazu greift das Werk Border as Method von Sandro Mezzadra und Brett Neilson (2013) zentral den Standpunkt des Ansatzes der Autonomie der Migration (s.u.) auf. Die Autoren definieren Grenzen als „social institutions, which are marked by tensions between practices of border reinforcement and border crossing“ (ebd., S. 3). Mit dem Begriff der „border struggles“ (ebd., S. 264ff) betonen sie die entscheidende Rolle der Kämpfe an und um Grenzen als wesentliches dynamisches Moment ihrer Konstitution bei der Bildung eines spezifischen Grenzregimes und dessen Inkraftsetzungen und Umsetzungen vor Ort.

Auch für die ethnografische Grenzregimeanalyse, wie wir sie anschließend darstellen werden, bildet die Mächtigkeit der Migration ihren Ausgangspunkt. Die Handlungsmacht der Migration als heuristischen Schlüssel für die Analyse von Grenzregimen zu verstehen, ist eine Perspektive, die wir - gemeinsam mit vielen Kolleg*innen - im Rahmen des Forschungszusammenhangs Transit Migration (2007) vor mehr als zehn Jahren entwickelt haben. Ausgehend vom „langen Sommer der Migration“ (Kasparek/Speer 2015) wollen wir diese Konzeptualisierung wieder aufgreifen, die mit Bezug auf den Ansatz der Autonomie der Migration Grenzräume als Produkt widerstreitender Kräfte, als emergentes Ergebnis des permanenten Ringens um Entkommen/Flucht einerseits und Einhegung andererseits versteht. Diese Perspektivierung ermöglicht Widerstandspraktiken der Bewegung der Migration, wie sie im Sommer 2015 offensichtlich geworden sind, aber auch grenzüberschreitende Praktiken ganz allgemein in eine Theorie der Grenze einzubringen, die den militarisierten Ausbau des Grenzregimes analytisch in den Blick nimmt und gleichzeitig der Migration als sozialer und politischer Macht gerecht wird. In dieser Perspektive des ethnografischen Grenzregimeansatzes ist das Grenzregime als ein Raum der ständigen Spannung, des Konflikts und der Anfechtung zu betrachten. 
Die Konzeptualisierung stellt einen methodologischen und theoretischen Versuch dar, nicht nur auf eine Art und Weise über die Beziehung zwischen Migrationsbewegungen und Kontrollregimen zu denken, die sich von der klassischen soziologischen Art und Weise der Objektstruktur unterscheidet. Sie führt auch zu einer anderen Vorstellung von Migration, als die bisherige vorherrschende Praxis in den Kultur- und Sozialwissenschaften es zuließ: sie nicht im Sinne einer ,Ableitung' vom Paradigma eines niedergelassenen Lebens in einem modernen Nationalstaat oder als eine funktionalistische Variable von wirtschaftlichen Prozessen und Rationalitäten zu sehen. Stattdessen versucht der Ansatz, Migration sowohl historisch als auch strukturell als einen Akt der Flucht und als ,unmerkliche‘ Form des Widerstandes im Sinne eines Sich-Entziehens zu konzeptualisieren, wie es von Dimitris Papadopoulos, Niamh Stephenson und Vassilis Tsianos (2008) beschrieben wird. Yann Moulier Boutang (2007) bezeichnete diesen Aspekt als „Autonomie der Migration“. Damit wird die Aufmerksamkeit auf die Migration als ko-konstituierenden Faktor der Grenze gerichtet, die mit den Kräften der Migrationsbewegungen, die die Grenze jeden Tag herausfordern und neuformieren, rechnen muss (vgl. Hess 2016b).

Doch was ändert sich, wenn wir Migration im Sinne des Konzepts der Autonomie der Migration denken? Das Konzept wird häufig falsch interpretiert, als wäre damit die Autonomie der Migrant*innen gemeint. Das verfehlt jedoch gänzlich seinen theoretischen Einsatz und seine Genese, da es vielmehr als strukturelles Argument aus einer historisch-materialistischen Interpretation der Geschichte verstanden werden muss. Auch beabsichtigt das Konzept nicht, das Leiden und die Notlage zahlreicher Migrationsprojekte zu verschleiern. Stattdessen stellt es einen Versuch dar, Migration wieder in die Geschichte der Arbeit, des Kapitalismus und der modernen Regierungsformen theoretisch und forschungspolitisch zu platzieren und damit die bisher oftmals ausgeblendete Fähigkeit der lebendigen Arbeit mitzudenken, den unerträglichen Verhältnissen der (Re-)Produktion zu entkommen (vgl. Mezzadra/Neilson 2013). Moulier Boutang (2007, S. 172) schreibt hierzu:

„Verknüpft man sie [die lebendige Arbeit] aber mit Foucaults, Wunsch der Massen, nicht auf diese Weise regiert zu werden', und schlägt den Bogen zum Konzept der Flucht oder des Exit[s], wird sie ergiebig. Denn Flucht ist die Weigerung der Massen, sich regieren zu lassen: eine Antwort auf asymmetrische Machtverhältnisse“.

In seinem Ansatz bezieht sich Moulier Boutang in erster Linie auf die theoretische Tradition des Operaismus (vgl. Pozzoli 1972; Negri 1977). Dieser entstand in den 1960er-Jahren in Italien einerseits als eine politische Bewegung, andererseits aber auch als eine politische Theorie im Widerspruch zum marxistischen Mainstream. Zwei zentrale Erkenntnisse des Operaismo scheinen für den Perspektivwechsel der Migrations- und Grenzforschung, wie er mit der These von der Autonomie der Migration einhergeht, entscheidend zu sein: zum einen die Konzeptualisierung der Geschichte des Kapitalismus als Ergebnis von Arbeiter*innen kämpfen. Unter diesem Gesichtspunkt erscheinen zum Beispiel sowohl die Industrialisierung als auch die Entwicklung der Fabrik als politische Antwort auf die massenhafte Flucht der arbeitenden Bevölkerung aus ländlichen Regionen und den Widerstand der Arbeitenden. Zum anderen sein Verständnis von Widerstand, wobei stille, unsichtbare, ungeordnete und scheinbar unbedeutende Formen der Subversion und des Sich-Entziehens, wie die bewusst verlangsamte Arbeitsverrichtung, ebenso als widerständige Akte berücksichtigt werden. Sinngemäß sieht Moulier Boutang die Ursache kapitalistischer Entwicklungen nicht nur in der Dynamik der Profitraten, sondern auch in den Reaktionen auf die gelebte Mobilität der Arbeitskräfte und im ständigen Versuch, 
lebendige Arbeit zu kontrollieren sowie deren Fähigkeit und Begehren, Widerstand zu leisten und den Verhältnissen zu entfliehen (vgl. Moulier Boutang 2007; Papadopoulos et al. 2008).

Diese Perspektive der Autonomie der Migration endet nicht mit der Annahme, dass Migration als eine aktive Kraft und als eine Form des alltäglichen stillen Widerstandes verstanden werden kann. Vielmehr fragt dieser Ansatz danach, wie Migration in das Zentrum der Wissensproduktion eingreift (vgl. Hess 2016b). Die Autonomie der Migration ist weniger ein Fazit als eine Perspektive, die neue Wege des Befragens und des Beforschens eröffnet. Oder, um Moulier Boutang (2007, S. 169) zu zitieren, sie „ist kein Slogan, sondern vielmehr eine Methode, ein Ausgangspunkt, ein heuristisches Modell, und nicht die Antwort auf eine Frage“.

\section{Die Autonomie der Migration als Prisma: methodologisch- theoretische Implikationen des Regimebegriffs}

Folgen wir dem Konzept der Autonomie der Migration als Methode oder als Prisma, stellt sich unweigerlich die Frage, was uns dieser Standpunkt für eine analytische Haltung ermöglicht. Erstens versteht der Ansatz Migration und Mobilität als soziale Bewegung, nicht im klassischen Sinne als geordnete, ideologisch angetriebene Bewegung, sondern eher als eine weltschaffende, kollektive Praxis und folglich als grundsätzlich politisches, soziales und transformatives Projekt. Der Vorschlag des Soziologen Asef Bayats (2010), sein Konzept der „nonmovements“ auf Migrationsbewegungen zu übertragen, setzt an diesem Verständnis von Migration an. Ausgehend von den gesellschaftlichen Kräfteverhältnissen in den postkolonialen Autokratien der 1980er-Jahre im Nahen Osten, referiert das Konzept auf die für die soziale Bewegungsforschung unkonventionellen Formen und Konturen sozialer Bewegungen. Nonmovements sind demnach "collective actions of noncollective actors“ (ebd., S. 14), die sich nicht unter einer gemeinsamen Ideologie oder Organisationen vereinen und stets fragmentiert bleiben. Doch aufgrund der Summe der jeweils vorgenommenen individuellen Handlungen, wie die informelle Errichtung von Camps oder das Anzapfen staatlicher Stromleitungen, stellen sie oftmals eine sukzessive Normalisierung jener „geteilten Praktiken“ her (ebd., S. 20f.). Sie können damit gesellschaftliche Transformationsprozesse auslösen, ohne dass sie von den Akteur*innen intendiert gewesen sein müssen. Bernd Kasparek und Matthias Schmidt-Sembdner (2017, S. 181) formulieren den Zusammenhang zwischen Bayats Konzept und der Perspektive der Autonomie der Migration mit Hinblick auf ihr gesellschaftliches Wirken:

„Dabei stellt Bayats Begriff der nonmovements und die Charakterisierung der Migration als soziale Bewegung in der Perspektive der Autonomie der Migration nur oberflächlich einen Widerspruch dar. Denn beiden semantischen moves geht es um das Insistieren auf einen sozialen und politischen Gehalt der analysierten gesellschaftlichen Phänomene. Bei Bayat bedeutet dies eine Abgrenzung von einem engen Bewegungsbegriff [...], während die Autonomie der Migration darauf besteht, dass Migration nicht nur eine räumliche Bewegung, sondern eben auch eine soziale Bewegung darstellt, die zu gesellschaftlichen Transformationen beiträgt“".

Zweitens, wenn wir mit dieser Perspektive auf die Grenze und auf das Migrationsregime schauen, ändert sich die Art und Weise, wie wir die Grenze konzeptualisieren und folglich auch unser Verständnis von Staat oder Souveränität. Der einst monolithische Grenzapparat zerbricht und zerfällt in viele einzelne Faktoren: Akteur*innen, Praktiken, Diskurse, Technologien, Körper, Emotionen, Prozesse und Kontroversen werden sichtbar, wobei die Migration als 
treibende Kraft fassbar wird (vgl. Heimeshoff et al. 2014, S. 13f.). Eine solche Konzeptualisierung der Grenze verwirft vereinfachte binäre Modelle, die Struktur in einem einfachen Gegensatz zu Handlungsmacht verorten. Stattdessen wird die Grenze als Raum der Herausforderung, des Konflikts und der Verhandlung neu konzeptualisiert. Die ethnografische Grenzregimeanalyse versucht eine methodologische Operationalisierung dieser theoretischen Implikationen zu bieten (vgl. Transit Migration Forschungsgruppe 2007; Tsianos/Hess 2010).

Sie baut auf den politikwissenschaftlichen Begriff des Regimes und dem Foucault'schen Verständnis (Foucault 1983; 2004; siehe auch Hess et al. 2018) auf, um borderwork als ein Gefüge aus einer Vielzahl von Akteur*innen, Institutionen und anderen menschlichen und nichtmenschlichen Faktoren und Praktiken zu betrachten, ohne die verschiedenen Interessen und Rationalitäten dieser Kräfte zu einer simplen linearen Logik oder einer versteckten Agenda zu vereinfachen (wie das des Kapitals oder europäischer Rassismus). Der Regimebegriff referiert auch auf die Komplexitäten und Widersprüche, ebenso wie auf Ad-hoc-Maßnahmen von Grenzpolitiken und ihrer Irrwege. Ebenso verweist er auf die Diskrepanzen zwischen Intention und Effekte migrationspolitischer Strategien und den umkämpften Handlungsparadigmen der verschiedenen beteiligten Akteur*innen. Dabei ist der Regimebegriff thematisch spezifisch angesetzt und prozesshaft orientiert. So schreibt auch Giuseppe Sciortino (2004, S. 32f.) über das Regime: „It is rather a mix of implicit conceptual frames, generations of turf wars among bureaucracies and waves after waves of ,quick fixes' to emergencies [...]: the life of a regime is the result of continuous repair work through practices".

Die ethnografische Grenzregimeanalyse basiert also eher auf einem empirischen und theoretischen Entwurf der Grenze als Gefüge, als Situation oder Site des kontinuierlichen Aufeinandertreffens und der Spannungen, an dem die Migration zu einem konstitutiven Bestandteil von Grenzziehungsprozessen wird. In der Folge ist mit dem Regimeansatz die kontinuierliche und strukturell konflikthafte Rekonfiguration der Grenze (als multiple verörtlichte und nichtörtliche, weitgespannte Assemblage) in erster Linie als eine Reaktion auf Migrationsbewegungen zu verstehen, die Grenzsituationen herausfordern, übertreten und umgestalten. Unter diesem Gesichtspunkt sind es die Migrationsbewegungen, die das soziopolitische und ökonomische Phänomen der Grenzräume erzeugen: Grenzräume sind das Produkt eines kollektivierten, überschüssigen Wunsches, Grenzen zu überwinden, von Netzwerken von Menschen in Bewegung und von kollektiven Wissenspraktiken der Grenzüberquerung (vgl. Fröhlich 2015).

Mit einem heuristischem Methodenmix aus symptomatischer Diskursanalyse, ethnografischer teilnehmender Beobachtung, informellen Gesprächen und teilstrukturierten sowie fokussierten, qualitativen Interviews schafft die ethnografische Grenzregimeanalyse ein Verständnis für die sozialen und politischen Prozesse des Grenzregimes in einem post-positivistischen und neokonstruktivistischem Sinne (vgl. Tsianos/Hess 2010, S. 252f.). Mit dem transversalen und diagonalen Ansatz des „studying through“ (vgl. Wright/Reinhold 2011) durchdringt die Grenzregimeanalyse auch das Mehrebenensystem der EU und zeigt die gaps zwischen einer written policy (z.B. in Form von Dokumenten der EU-Kommission) und den alltäglichen Praktiken der untersuchten Akteur*innen in den Grenzräumen sowie ihre wechselseitigen Beeinflussungen.

\section{Radikalisierung, Ausweitung, Stagnation: das europäische Grenzregime nach dem Sommer der Migration}

Mit diesem empirischen Forschungsansatz, der die Grenze als Konflikt- und Aushandlungszonen ethnografisch erfasst, lässt der (noch unabgeschlossene) Restabilisierungsprozess des 
Grenzregimes in den vergangenen vier Jahren keinen radikalen Bruch mit den bisherigen Paradigmen erkennen. Gleichwohl ist er durch neue Konturen und Dynamiken gekennzeichnet: Er enthält Züge der Radikalisierung, Ausweitung und Verlagerung ebenso wie des Rückbaus mancher Elemente und der Stagnation. Mit dem EU-Türkei-Deal und der erneuerten Einbindung Libyens zur Unterbindung der Migration über die zentrale Mittelmeerroute nach Europa bleiben Strategien der Externalisierung dominant. Mit der Aufrüstung von Grenzen durch manifeste Architekturen werden die smarten und intelligenten Technologien der Überwachung und Migrationssteuerung wieder um sehr materielle Aspekte ergänzt und teils durch sie ersetzt. Eine solche Rematerialisierung der Grenze kann als hardening of the border beschrieben werden. Neben dem durch die Dublin-Verordnung institutionalisierten internen Regime tritt auch eine verstärkte Reterritorialisierung in Form einer Renationalisierung des Schengenraums: Die sukzessive Wiedereinführung nationaler Grenzkontrollen in weiten Teilen des Binnenraums, beginnend im Herbst 2015, verlagerte beziehungsweise erweiterte die Kontrolle und Unterbindung der sogenannten „Sekundärmigration“ von den Asylbehörden an die jeweiligen Staatsgrenzen (vgl. Schmidt-Sembdner 2018). Zeitgleich erweist sich das Dublin-System als nahezu reform-resistent und steht seit Jahren exemplarisch für die festgefahrenen Verhandlungen der EU-Länder in der Frage der Verteilung von Asylsuchenden in Europa (vgl. Kasparek 2018). Die „humanitäre Grenze“ im Sinne Walters (2011) ist zunehmend in die Defensive geraten und wurde durch manifeste anti-humanitaristische Diskurse und Politiken zumindest medial in den Schatten gestellt. Informelle, illiberale und auch illegale Praktiken, sei es im Zusammenhang mit Aufnahmeprozeduren in den Hot-Spot-Centern entlang der Außengrenze oder mit Blick auf die Push-Back-Politiken auf See wie auf Landwegen, haben den Prozess der Verrechtlichung des Grenzregimes wieder zurückgedrängt. So müssen wir heute eher von einer „nekropolitischen Grenze“ (Mbembe 2003) sprechen, welche die Politik des Sterben-Lassens im Mittelmeer genauso umfasst wie die systemische direkte, interpersonale Gewalt in den libyschen Lagern oder den EU-Grenzräumen auf dem Balkan.

\section{Weiterführende Literatur}

Hess, Sabine/Kasparek, Bernd/Kron, Stefanie/Rodatz, Mathis/Schwertl, Maria/Sontonwski, Simon (Hrsg.) (2016): Grenzregime III. Der lange Sommer der Migration. Berlin: Assoziation A.

Mezzadra, Sandro/ Neilson, Brett (2013): Border as Method, or, the Multiplication of Labor. Durham/London: Duke University Press.

Pott, Andreas/Rass, Christoph/Wolff, Frank (Hrsg.) (2018): Was ist ein Migrationsregime? What is a migration regime? Wiesbaden: Springer VS.

Riedner, Lisa (2016): Arbeit! Wohnen! Urbane Auseinandersetzungen um EU-Migration. Eine Untersuchung zwischen Wissenschaft und Aktivismus. Münster: edition assemblage.

Transit Migration Forschungsgruppe (Hrsg.) (2007): Turbulente Ränder. Neue Perspektiven auf Migration an den Grenzen Europas. Bielefeld: transcript.

\section{Literaturverzeichnis}

Andreas, Peter (2000): Introduction: The Wall after the Wall. In: Ders./Snyder, Timothy (Hrsg.): The Wall around the West: State Borders and Immigration Controls in North America and Europe. Lanham/New York/Oxford: Rowman \& Littlefield, S. 1-14.

Andreas, Peter/Snyder, Timothy (Hrsg.) (2000): The Wall around the West: State Borders and Immigration Controls in North America and Europe. Lanham/New York/Oxford: Rowman \& Littlefield.

Balibar, Étienne (2002): Politics and the Other Scene. London: Verso.

Balibar, Étienne (1997/2006): Der Schauplatz des Anderen. Formen der Gewalt und Grenzen der Zivilität. Hamburg: Hamburger Edition.

Barth, Frederik (1969/1998): Ethnic Groups and Boundaries: The Social Organization of Culture Difference. Long Grove: Waveland Press.

Bayat, Asef (2010): Life as Politics. How ordinary people change the Middle East. Stanford: Stanford University Press. 
Bialasiewicz, Luiza (2012): Off-Shoring and Out-Sourcing the Borders of Europe: Libya and EU Border Work in the Mediterranean. In: Geopolitics 17, H. 4, S. 843-866.

Bigo, Didier/Guild, Elspeth (2005): Policing in the Name of Freedom. In: Dies. (Hrsg.): Controlling Frontiers: Free Movement into and within Europe. Aldershot: Ashgate, S. 1-13.

Bojadžijev, Manuela (2006): Das ,Spiel‘ der Autonomie der Migration. In: ZfK - Zeitschrift für Kulturwissenschaften 1, S. 139-145.

Borri, Giulia/ Fontanari, Elena (2016): Lampedusa in Berlin. (Im)Mobilität innerhalb des Europäischen Grenzregimes. In: Peripherie 35, H. 138/139, S. 193-211.

Brambilla, Chiara (2015): Exploring the Critical Potential of the Borderscapes Concept. In: Geopolitics 20, H. 1. S. 14-34.

Broeders, Dennis (2007): The New Digital Borders of Europe: EU Databases and the Surveillance of Irregular Migrants. In: International Sociology 22, H. 1, S. 71-92.

Butler, Judith (1991): Das Unbehagen der Geschlechter. Frankfurt/M.: Suhrkamp.

Carrera, Sergio/Hertog, Leonhard den (2015): Whose Mare? Rule of Law Challenges in the Field of European Border Surveillance in the Mediterranean. In: Centre for European Policy Studies (CEPS) Papers in Liberty and Security in Europe 79, S. 1-29.

Commission of the European Communities (2008): Preparing the Next Steps in Border Management in the European Union. In: Communication from the Commission to the European Parliament, the Council, the European Economic and Social Committee and the Committee of the Regions. Brüssel.

Cuttitta, Paolo (2016): Zwischen De- und Repolitisierung. Nichtstaatliche Search and Rescue-Akteure an der EU-Mittelmeergrenze. In: Hess, Sabine/Kasparek, Bernd/Kron, Stefanie/Rodatz, Mathis/Schwertl, Maria/Sontonwski, Simon (Hrsg.): Grenzregime III. Der lange Sommer der Migration. Berlin: Assoziation A, S. 115-125.

Dijstelbloem, Huub/Meijer, Albert/Besters, Michiel (2011): The Migration Machine. In: Dies. (Hrsg.): Migration and the New Technological Borders of Europe. London: Palgrave Macmillan, S. 1-22.

Donnan, Hastings/Wilson, Thomas M. (2010): Borderlands: Ethnographic Approaches to Security, Power, and Identity. Lanham/New York/Oxford: Rowman \& Littlefield.

Fassin, Didier (2007): Humanitarianism as a Politics of Life. In: Public Culture 19, H. 3, S. 499-520.

Foucault, Michel (1983): Sexualität und Wahrheit I: Der Wille zum Wissen. Frankfurt/M.: Suhrkamp.

Foucault, Michel (2004): Geschichte der Gouvernementalität I. Sicherheit, Territorium, Bevölkerung. Vorlesung am Colleges de France 1977/1978. Frankfurt/M.: Suhrkamp.

François, Etienne/Seifarth, Jörg/ Struck, Bernhard (Hrsg.) (2007): Die Grenze als Raum, Erfahrung und Konstruktion: Deutschland, Frankreich und Polen vom 17. bis 20. Jahrhundert. Frankfurt/M./New York: Campus.

Fröhlich, Marie (2015): Routes of Migration - Migrationsprojekte unter Bedingungen europäisierter Regulation. In: Hess, Sabine/Näser, Torsten (Hrsg.): Movements of Migration: Neue Perspektiven im Feld von Stadt, Migration und Repräsentation. Berlin: Panama-Verlag, S. 150-162.

Glick Schiller, Nina/Salazar, Noel B. (2013): Regimes of Mobility Across the Globe. In: Journal of Ethnic and Migration Studies 39, S. 183-200.

Hall, Stuart/Gieben, Bram (1992): Formations of Modernity. Cambridge: Polity Press/Open University.

Heimeshoff, Lisa-Marie/Hess, Sabine/Kron, Stefanie/Schwenken, Helen/Trzeciak, Miriam (2014): Einleitung. In: Dies. (Hrsg.): Grenzregime II: Migration - Kontrolle - Wissen. Transnationale Perspektiven. Berlin/Hamburg: Assoziation A, S. 5-26.

Hess, Sabine (2016a): „Citizens on the Road“: Migration, Borders, and the Reconstruction of Citizenship in Europe. In: JEECA. Journal of European Ethnology and Cultural Analysis 1, S. 7-22.

Hess, Sabine (2016b): Migration als widerständige Praxis - Die Autonomie der Migration als theoretische Intervention in die Border Studies. In: Dülcke, Dana/Kleinschmidt, Julia/Tietje, Olaf/Wenke, Juliane (Hrsg.): Grenzen von Ordnung. Eigensinnige Akteur_innen zwischen (Un)Sicherheit und Freiheit. Münster: Verlag Westfälisches Dampfboot.

Hess, Sabine/Karakayali, Serhat (2007): New Governance oder die imperiale Kunst des Regierens. In: Transit Migration Forschungsgruppe (Hrsg.): Turbulente Ränder. Neue Perspektiven auf Migration an den Grenzen Europas. Bielefeld: transcript, S. 39-56.

Hess, Sabine/Tsianos, Vassilis (2007): Europeanizing Transnationalism! Provincializing Europe! - Konturen eines Neuen Grenzregimes. In: Transit Migration Forschungsgruppe (Hrsg.): Turbulente Ränder. Neue Perspektiven auf Migration an den Grenzen Europas. Bielefeld: transcript, S. 23-38.

Hess, Sabine/Kasparek, Bernd (2017): De- and Restabilising Schengen: The European Border Regime after the Summer of Migration. In: Cuadernos Europeos de Deusto: Governing Mobility in Europe: Interdisciplinary Perspectives 56, S. 47-78.

Hess, Sabine/Kasparek, Bernd/Schwertl Maria (2018): Regime ist nicht Regime ist nicht Regime. Zum theoriepolitischen Einsatz der ethnographischen (Grenz-)Regimeanalyse. In: Pott, Andreas/Rass, Chris- 
toph/Wolff, Frank (Hrsg): Was ist ein Migrationsregime? What is a migration regime? Wiesbaden: Springer VS, S. 257-284.

Houtum, Henk van/Naerssen, Ton van (2002): Bordering, Ordering, Othering. Tijdschrift voor economische en sociale geografie 93, S. 125-136.

Huysmans, Jef (2000): The European Union and the Securitization of Migration. In: Journal of Common Market Studies 33, S. 751-777.

Karakayali, Serhat (2008): Gespenster der Migration. Zur Genealogie illegaler Einwanderung in der Bundesrepublik Deutschland. Bielefeld: transcript.

Karamanidou, Lena/Kasparek, Bernd (2018): Border Management and Migration Control in the European Union. In: Respond Working Papers: Global Migration: Consequences and Responses 14.

Kasparek, Bernd (2016): Complementing Schengen: The Dublin System and the European Border and Migration Regime. In: Bauder, Harald/Matheis, Christian (Hrsg.): Migration Policy and Practice. London: Palgrave Macmillan, S. 59-78.

Kasparek, Bernd (2018): Abschottung im Recht, digitale Erfassung und forcierte Europäisierung. Das kommende Grenzregime nach den Plänen der Europäischen Kommission. Berlin: Rosa-Luxemburg-Stiftung,

Kasparek, Bernd/Speer, Marc (2015): Of hope. Ungarn und der lange Sommer der Migration. https://borde rmonitoring.eu/ungarn/2015/09/of-hope/, 17.11.2019.

Kasparek, Bernd/Schmidt-Sembdner, Matthias (2017): Towards Democracy. Die Bewegung der Migration und die Demokratisierung des europäischen Projektes. In: Candeias, Mario/Demirović, Alex (Hrsg.): Europe - what's left? Die Europäische Union zwischen Zerfall, Autoritarismus und demokratischer Erneuerung. Münster: Westfälisches Dampfboot, S. 175-191.

Kearney, Michael (1991): Borders and Boundaries of State and Self at the End of Empire. In: Journal of Historical Sociology 4, H. 1, S. 52-74.

Koslowski, Rey (2005): Smart Borders, Virtual Borders or No Borders: Homeland Security Choices for the United States and Canada. www.albany.edu/ rk289758/documents/Koslowski_Smart_Borders_SMU_la w_Review05.pdf, 06.08.2019

Kuster, Brigitta/Tsianos, Vassilis (2014): Zur Digitalisierung der europäischen Grenze. Der Fall Eurodac. In: Cilip: Bürgerrechte und Polizei 105, S. 61-68.

Lahav, Gallya/Virginie Guiraudon (2000): Comparative Perspectives on Border Control: Away from the Border and Outside the State. In: Andreas, Peter/Snyder, Timothy (Hrsg.): The Wall Around the West: State Borders and Immigration Controls in North America and Europe. Lanham/New York/Oxford: Rowman \& Littlefield, S. 55-77.

Laine, Jussi (2016): The Multiscalar Production of Borders. In: Geopolitics 21, H. 3. S. 465-482.

Lavenex, Sandra (2004): EU External Governance in „Wider Europe“. In: Journal of European Public Policy 11, H. 4, S. 680-700.

Lemke, Thomas (2007). Biopolitik zur Einführung. Hamburg: Junius.

Mbembe, Achille (2003): Necropolitics. In: Public Culture 15, H. 1, S. 11-40.

Mezzadra, Sandro/Neilson, Brett (2013). Border as Method, or, the Multiplication of Labor. Durham/London: Duke University Press.

Morris, Lydia (2002): Managing Migration: Civic Stratification and Migrants Rights. London/New York: Routledge Chapman \& Hall.

Moulier Boutang, Yann (2007): Europa, Autonomie der Migration, Biopolitik. In: Pieper, Marianne/Atzert, Thomas/Karakayali, Serhat/Tsianos, Vassilis (Hrsg.): Empire und die biopolitische Wende. Die internationale Diskussion im Anschluss an Hardt und Negri. Frankfurt/M.: Campus, S. 169-180.

Negri, Tony (1997): Massenautonomie gegen Historischen Kompromiß. München: Trikont.

Newman, David (2006). The Lines that Continue to Separate Us: Borders in a Borderless World. In: Progress in Human Geography 30, H. 2, S. 143-161.

Ohmae, Kenichi (1990). The Borderless World: Management Lessons in the New Logic of the Global Market Place. London: Collins.

Pallister-Wilkins, Polly (2017): Humanitarian borderwork: actors, spaces, categories. In: Political Geography 59, S. 5-7.

Papadopoulos, Dimitris/Stephenson, Niamh/Tsianos, Vassilis (2008): Escape Route: Control and Subversion in the Twenty-first Century. London: Pluto.

Parker, Noel/Vaughan-Williams, Nick (2009): Lines in the Sand? Towards an Agenda for Critical Border Studies. In: Geopolitics 14, H. 3, S. 582-587.

Pozzoli, Claudio (Hrsg.) (1972): Spätkapitalismus und Klassenkampf: eine Auswahl aus den Quaderni Rossi. Frankfurt/M.: Europäische Verlagsanstalt.

Rumford, Chris (2006): Theorizing Borders. In: European Journal of Social Theory 9, H. 2, S. 155-169. 
Rumford, Chris (2008): Introduction: Citizens and Borderwork in Europe. In: Space and Polity 12, H. 1, S. $1-12$.

Salter, Mark B (2011): Places Everyone! Studying the Performativity of the Border. In: Political Geography 30, S. 66-67.

Schmidt-Sembdner, Matthias (2018): Grenzkontrollen als „dauerhaftes Provisorium“? Renationalisierungsprozesse im Schengenraum am Beispiel der Brennerroute. In: movements. Journal for Ciritcal Migration and Border Regime Studies 4, H. 2, S. 57-76.

Schmidt-Sembdner, Matthias (2019): Europa und die Bewegung der Migration. Das Dublin-System im Kontext nationaler Grenzkontrollen entlang der Brenner-Route. In: Johler, Reinhard/Lange, Jan (Hrsg.): Konfliktfeld Fluchtmigration. Historische und Ethnographische Perspektiven. Bielefeld: transcript, S. 249-264.

Schuster, Liza (2011): Dublin II and Eurodac: Examining the (Un)intendend(?) Consequences. In: Gender, Place \& Culture: A Journal of Feminist Geography 18, H. 3, S. 401-416.

Sciortino, Guiseppe (2004): Between Phantoms and Necessary Evils: Some Critical Points in the Study of Irregular Migrations to Western Europe. In: Böcker, Anita/de Hart, Betty/Michalowski, Ines (Hrsg.): Migration and the Regulation of Social Integration. Osnabrück: University of Osnabrück, Institut für Migrationsforschung und Interkulturelle Studien (IMIS), S. 17-43.

Transit Migration Forschungsgruppe (2007): Turbulente Ränder. Neue Perspektiven auf Migration an den Grenzen Europas. Bielefeld: transcript.

Tsianos, Vassilis/Hess, Sabine (2010): Ethnographische Grenzregimeanalyse. In: Hess, Sabine/Kasparek, Bernd (Hrsg.): Grenzregime: Diskurse, Praktiken, Institutionen in Europa. Assoziation A, S. 243-264.

Walters, William (2002): Mapping Schengenland: Denaturalizing the border. In: Environment and Planning D: Society and Space 20, H. 5, S. 561-580.

Walters, William (2006): Border/Control. In: European Journal of Social Theory 9, H. 2, S. 187-203.

Walters, William (2011): Foucault and Frontiers: Notes on the Birth of the Humanitarian Border. In: Krasmann, Ulrich/Lemke, Thomas (Hrsg.): Governmentality: Current Issues and Future Challenges. New York: Routledge, S. 138-164.

Walters, William/Haahr, Jens Henrik (2004): Governing Europe: Discourse, Governmentality and European Integration. New York: Routledge.

Wright, Susan/Reinhold, Sue (2011): „Studying Through“: A Strategy for Studying Political Transformation. Or Sex, Lies and British Politics. In: Shore, Chris/Wright, Susan/Però, Davide (Hrsg.): Policy Worlds: Anthropology and the Analysis of Contemporary Power. New York: Berghahn Books, S. 86-104.

Zolberg, Aristide R. (2006): Managing a World on the Move. In: Population and Development Review 32 , H. 1, S. 222-253. 